\title{
Cantharidin suppresses cell growth and migration, and activates autophagy in human non-small cell lung cancer cells
}

\author{
YAN-PENG LIU ${ }^{1}$, LING LI ${ }^{2}$, LIANG XU ${ }^{2}$, E-NUO DAI ${ }^{2}$ and WEI-DA CHEN ${ }^{3}$ \\ ${ }^{1}$ Department of Internal Medicine, The Second Hospital of Shandong University, Jinan, Shandong 250033; \\ ${ }^{2}$ Department of Surgery, Affiliated Hospital of Shandong Academy of Medical Sciences, Jinan, Shandong 250031; \\ ${ }^{3}$ Department of Geriatric Medicine, Affiliated Hospital of Shandong University of Traditional Chinese Medicine, \\ Jinan, Shandong 250014, P.R. China
}

Received June 16, 2017; Accepted December 5, 2017

DOI: $10.3892 / \mathrm{ol} .2018 .8141$

\begin{abstract}
Cantharidin (CTD), a component of Mylabris (blister beetle), is a traditional Chinese medicine that exerts an anticancer effect in multiple types of cancer cells. The aim of the present study was to investigate whether CTD exhibited anti-metastatic and inhibitory cell proliferation effects against human non-small cell lung cancer (NSCLC) A549 cells, and the possible underlying mechanism by which this occurs. The results of the present study demonstrated that CTD arrested proliferation, suppressed invasion and migration and induced apoptosis in A549 cells in vitro. Alterations of apoptosis-associated protein levels, including B-cell lymphoma-2 (Bcl-2), Bcl-associated X (Bax) and active caspase-3, were detected. Furthermore, the present study demonstrated that CTD activated autophagy through downregulation of p62 expression and upregulation of microtubule-associated proteins 1A/1B light chain 3B and Beclin-1 expression. Additionally, western blot analysis identified that CTD inhibited the phosphatidylinositol 3-kinase (PI3K)/RAC serine/threonine protein kinase $(\mathrm{Akt}) /$ mechanistic target of rapamycin (mTOR) signaling pathway in NSCLC, demonstrating that the levels of phosphorylated (p-)Akt, p-mTOR, phosphorylated ribosomal p70S6 protein kinase (p-p70-S6K) and cyclin D1 were significantly decreased following treatment with CTD. In conclusion, the results of the present study indicated that CTD impeded cell growth and migration by inhibiting PI3K/Akt/mTOR signaling in NSCLC, and promoted autophagy and apoptosis. CTD exhibited anticancer activity against NSCLC in vitro, revealing it as a potential candidate for the treatment of NSCLC.
\end{abstract}

Correspondence to: Dr Yan-Peng Liu, Department of Internal Medicine, The Second Hospital of Shandong University, 247 North Park Avenue, Jinan, Shandong 250033, P.R. China

E-mail: lyp_327@163.com

Key words: cantharidin, autophagy, non-small cell lung cancer cell, proliferation, phosphoinositide 3-kinase/RAC serine/threonine-protein kinase/mechanistic target of rapamycin signaling pathway

\section{Introduction}

Lung cancer is a malignant type of cancer with a high incidence rate in China; it is primarily classified into two groups, small cell lung carcinoma and non-small cell lung carcinoma (NSCLC). Approximately $80 \%$ of patients with lung cancer have NSCLC $(1,2)$, furthermore, $>50 \%$ of these patients present with advanced local invasion and distant metastasis (3). Although chemotherapy is the principal treatment modality for the majority of patients, it is associated with a series of detrimental side effects, including suppression of the medulla oblongata, impaired immune function and toxicity in other organs (4-6). Therefore, improved therapeutic strategies and novel drug targets for NSCLC are required.

Autophagy is an important catabolic cellular homeostatic process; its mechanism involves the degradation of abnormal or dysfunctional cellular components resulting from digestion in lysosomes, which is associated with survival, differentiation and development in the normal physiology of cells (7). A number of studies have previously reported that autophagy exerts dynamic effects, including the promotion of apoptosis and the inhibition of proliferation in tumor cells (8-10). During the initiation of autophagy, Beclin-1 is able to promote LC3 to convert it to LC3-II, which is recruited to the major markers closely associated with autophagy are LC3 and p62. P62 binds to autophagosomal membrane and has been widely used as a protein marker to indicate the occurrence of autophagy. Two LC3 via the LC3 interacting region domain and is then degraded during the autophagy process. Thus, the conversion of LC3 I to LC3 II and clearance of p62 are considered hallmarks of the autophagic flux $(11,12)$. However, the underlying molecular mechanisms of autophagy involved in cancer occurrence and development remain unresolved.

Therefore, drugs targeting autophagy may serve as a therapeutic strategy for patients with NSCLC. It has been reported that Cantharidin (CTD), an active chemical compound isolated from the blister beetle (Coleoptera: Meloidae), serves a notable role in promoting autophagy and suppressing hepatocellular carcinoma (13). Consequently, one aim of the present study was to investigate the association between CTD and autophagy. A further aim of present study was to characterize the antitumor effect of CTD, which mediated the inhibition of metastasis and 
growth by and its possible underlying mechanism in NSCLC using A549 cells.

Therefore, the present study aimed to investigate the effect of CTD on NSCLC cell proliferation and metastasis and explore the potential molecular mechanism, which may aid in identifying a novel agent for NSCLC therapy.

\section{Materials and methods}

Chemicals and antibodies. CTD was purchased from MedChemExpress (Monmouth Junction, NJ, USA). Primary antibodies against rabbit active caspase-3 (\#9661), rabbit RAC serine/threonine-protein kinase (AKT; \#9272), rabbit phosphorylated-(p-)AKT: (\#9271), rabbit mechanistic target of rapamycin (mTOR; \#2972), rabbit p-mTOR: (\#2971), rabbit phosphorylated p-ribosomal p70S6 protein kinase (p-p70S6K; \#9209) were purchased from Cell Signaling Technology, Inc. (Danvers, MA, USA). Primary antibodies against rabbit B cell lymphoma (Bcl)-2 (12789-1-AP), rabbit Bcl-2-associated X protein (Bax; 50599-2-Ig), mouse cyclin D1 (60186-1-Ig), rabbit Microtubule-associated protein 1A/1B-light chain 3 (LC3; 14600-1-AP), rabbit Beclin-1 (11306-1-AP), rabbit p62 (18420-1-AP), mouse GAPDH (60004-1-Ig), anti-rabbit (15134-1-AP) or anti-mouse (30000-0-AP) IgG-horseradish peroxidase-conjugated antibodies and the enhanced chemiluminescence (ECL) detection system were purchased from ProteinTech Group, Inc. (Wuhan, China).

Cell culture. The human lung cancer A549 cell line was purchased from the Institute of Biochemistry and Cell Biology (Shanghai, China) and were cultured at $37^{\circ} \mathrm{C}$ in $5 \%$ $\mathrm{CO}_{2}$ in RPMI-1640 medium supplemented with $10 \%$ FBS (both GE Healthcare Bio-Sciences, Pittsburgh, PA, USA), $100 \mathrm{U} / \mathrm{ml}$ penicillin $\mathrm{G}$ and $100 \mu \mathrm{g} / \mathrm{ml}$ streptomycin sulfate (Sigma-Aldrich; Merck KGaA, Darmstadt, Germany). Experiments were conducted with cells in the logarithmic growth phase $\left(0.5-1 \times 10^{6}\right.$ cells $\left./ \mathrm{ml}\right)$. The experimental groups were treated with $1 \mu \mathrm{MCTD}$, which was in accordance with an effective minimum concentration as investigated by previous studies $(14,15)$; the negative control group (NC) was cultured with $1 \%$ dimethyl sulfoxide (DMSO) culture media.

Cell proliferation assay. Cell proliferation was evaluated using the Cell Counting kit-8 (CCK-8) (Beijing Solarbio Science and Technology Co., Ltd., China) in accordance with the manufacturer's protocol. A total of $1 \times 10^{3}$ cells/well were seeded into 96-well plates for $24 \mathrm{~h}$ and then were incubated with CTD $(1 \mu \mathrm{M})$ and a control group (1\% DMSO in culture RPMI-1640 medium) for 24, 48 or $72 \mathrm{~h}$. Absorbance (optical density) of viable cells was measured at a wavelength of $450 \mathrm{~nm}$ using a microplate reader (Bio-Rad Laboratories, Inc., Hercules, CA, USA).

Cell migration and invasion assay. Following treatment of cells with CTD for $48 \mathrm{~h}$, cell migration and invasion were evaluated using 24-well transwell chambers (EMD Millipore, Billerica, MA, USA), in the presence (invasion assay) and absence (migration assay) of Matrigel matrix (BD Biosciences, Franklin Lakes, NJ, USA), according to the manufacturer's protocol. A total of $1 \times 10^{5}$ cells were resuspended in $100 \mu 1$ serum-free

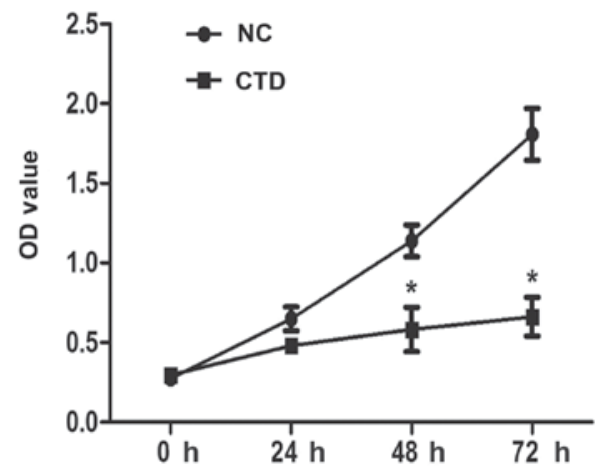

Figure 1. CTD attenuates the growth of A549 human lung cancer cells. A549 cells were treated with $1 \mu \mathrm{M}$ CTD for $0,24,48$ and $72 \mathrm{~h}$ and the cell viability was subsequently analyzed by Cell Counting kit- 8 assay. Data are presented as the mean \pm standard deviation. ${ }^{*} \mathrm{P}<0.05$ vs. NC groups. CTD, cantharidin; OD, optical density.

RPMI-1640 medium and seeded in the upper chambers. The lower chambers were filled with $500 \mu$ l RPMI-1640 medium with 10\% FBS (GE Healthcare Life Sciences, Shanghai, China). Following incubation at $37^{\circ} \mathrm{C}$ for $24 \mathrm{~h}$, non-invading cells were removed using a cotton-tipped swab. The cells that permeated through the membrane to the bottom chamber were fixed at room temperature in $4 \%$ paraformaldehyde for $10 \mathrm{~min}$ and then stained with $0.5 \%$ crystal violet for $15 \mathrm{~min}$ at room temperature. The number of invaded or migrated cells was quantified by counting five random fields for each membrane under an inverted microscope (Olympus, Tokyo, Japan) with 10x20 magnification and the average number of cells per field was calculated.

Cell apoptosis analysis. An Annexin V-Fluorescein Isothiocyanate (FITC)/propidium iodide (PI) Apoptosis Detection kit (Beijing Chemclin Biotech Co. Ltd., Beijing, China) was used to detect apoptosis according to the manufacturer's protocol. Cells were incubated with $1 \mu \mathrm{M}$ CTD at $37^{\circ} \mathrm{C}$ for $24 \mathrm{~h}$ and then changed to a culture with serum-free RPMI-1640 medium for $24 \mathrm{~h}$ and cells were harvested by trypsinization without EDTA (Invitrogen; Thermo Fisher Scientific, Inc., Waltham, MA, USA). Cells were resuspended in $1 \mathrm{X}$ binding buffer at $1-5 \times 10^{6} \mathrm{cell} / \mathrm{s} / \mathrm{ml}$. A total of $5 \mu \mathrm{l}$ annexin V-FITC was added to $100 \mu \mathrm{l}$ cell suspension and incubated in the dark for $5 \mathrm{~min}$ at room temperature. Following this, $10 \mu 1$ PI and $500 \mu 1$ PBS were added. The samples were then analyzed using a FACSCalibur cytometer (BD Biosciences) within $1 \mathrm{~h}$. CellQuest Pro (version 5.1; BD Biosciences) was used for data analysis.

Western blot analysis. CTD groups and NC groups of A549 cells were incubated with their respective treatments for $24 \mathrm{~h}$. Total protein was extracted from cells with radioimmunoprecipitation assay buffer (Cell Signaling Technology) containing protease and phosphatase inhibitor cocktails (Sigma-Aldrich; Merck KGaA) and the BCA Protein assay kit (Beijing ComWin Biotech Co., Ltd, Beijing, China) was used to determine the protein concentration. Equal quantities of proteins $(20 \mu \mathrm{g})$ were separated by $8-12 \%$ SDS-PAGE and transferred to a polyvinylidene difluoride membrane (EMD Millipore) for immunoblotting analysis. Then, membranes were blocked 
A

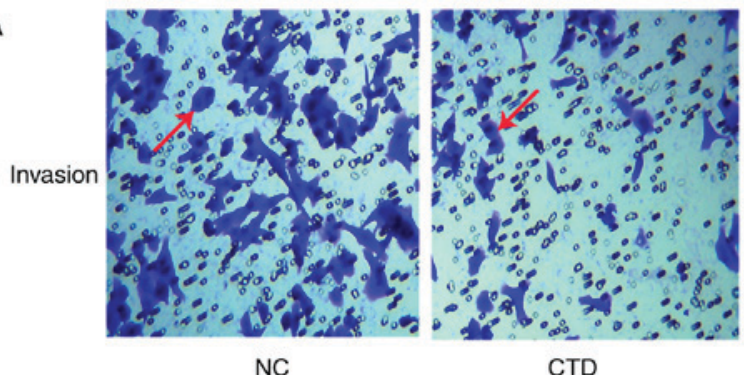

NC

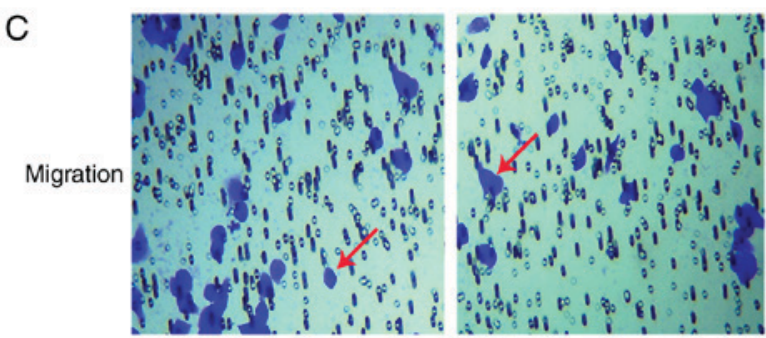

$\mathrm{NC}$

CTD
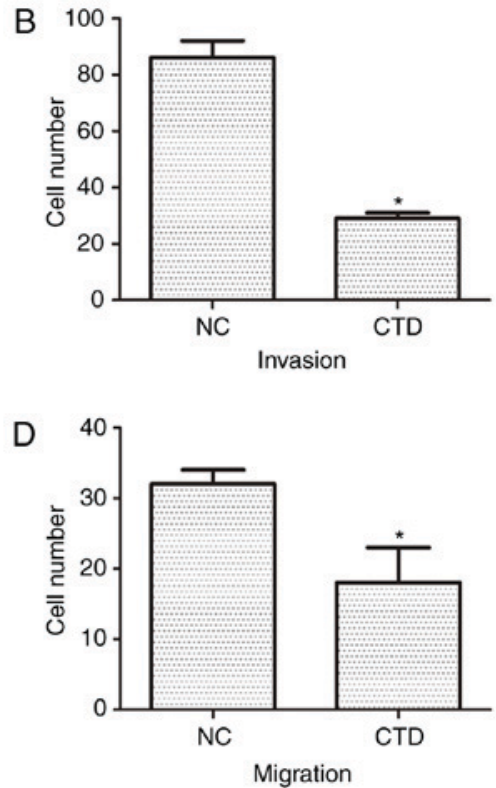

Figure 2. CTD represses cells invasion and migration in vitro. (A) Images demonstrating the invasion of CTD-treated A549 cells under an inverted microscope with 200x magnification. (B) Quantification of invaded CTD-treated A549 cells. (C) Images depicting the migration of CTD-treated A549 cells under an inverted microscope with 200x magnification. (D) Quantification of migrated CTD-treated cells. Data are presented as mean \pm SD. * $<<0.05$ vs. NC group. The red arrows indicate cells stained by $0.5 \%$ crystal violet as invading or migrating cells. All data are presented as the mean \pm standard deviation. CTD, cantharidin; NC, negative control.

with $5 \%$ non-fat milk at room temperature for $1.5 \mathrm{~h}$. The membranes were subsequently incubated with primary antibodies against cyclin D1 (dilution 1:1,000), p-p70S6K (dilution 1:1,000 dilution), Bcl-2 (dilution 1:1,000), Bax (dilution 1:1,000), active caspase-3 (dilution 1:1,000), LC3 (dilution 1:1,000), Beclin-1 (dilution 1:1,000), p62 (dilution 1:1,000) and GAPDH (for reference; dilution 1:5,000) (ProteinTech Group, Inc., Wuhan, Sanying, China) at $4^{\circ} \mathrm{C}$ overnight followed by the aforementioned horseradish peroxidase-conjugated secondary antibodies (dilution 1:5,000) at room temperature in darkness for $2 \mathrm{~h}$ and developed with the aforementioned ECL method (ProteinTech Group, Inc.) in accordance with the manufacturers protocol. Densitometry analysis was performed using Image-Pro Plus 6.0 software (Media Cybernetics Inc. Rockville, MD, USA).

Statistical analysis. SPSS 22.0 (IBM Corp., Armonk, NY, USA) and GraphPad Prism 5 (GraphPad Software, Inc., La Jolla, CA, USA) were used to perform statistical analysis. Data are expressed as the mean \pm standard deviation. The differences between the two groups were analyzed by Student's t-test. All experiments were performed in triplicate. $\mathrm{P}<0.05$ was considered to indicate a statistically significant difference.

\section{Results}

CTD attenuates the growth, invasion and migration of A549 cells. The effect of CTD on proliferation of A549 cells was determined by CCK-8 assay. Results demonstrated that CTD significantly inhibited the proliferation of A549 cells at 48 and $72 \mathrm{~h}$, following treatment with $1 \mu \mathrm{M}$ CTD (Fig. $1, \mathrm{P}<0.05$ ).

To confirm the effect of CTD on the migration and invasion of A549 cells, transwell invasion and migration assays were conducted. As Fig. 2A depicts, the number of invading cells was
A
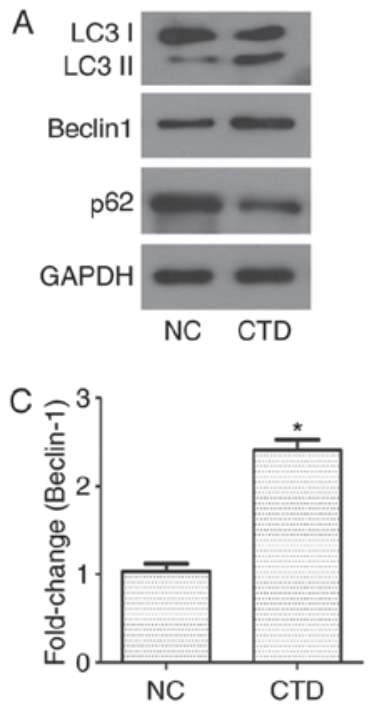
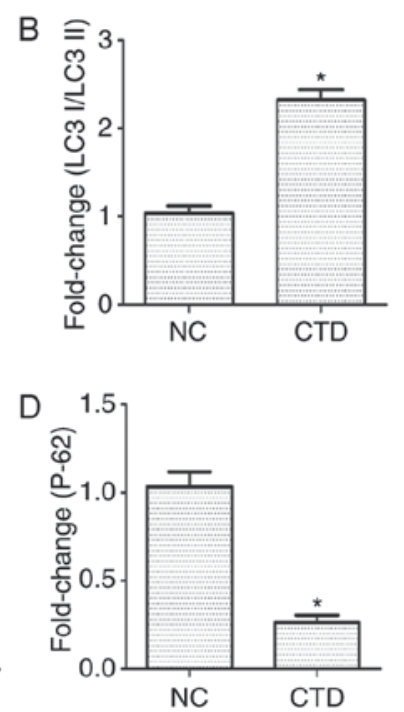

Figure 3. CTD enhances A549 cells autophagy. (A) Western blotting of autophagy-associated proteins LC3 I , LC3 II, Beclin-1 and p62 were analyzed by. (B-D) Quantifications of (B) LC3 I/LC3 II, (C) Beclin-1 and (D) p62. All data are presented as the mean \pm standard deviation. ${ }^{*} \mathrm{P}<0.05$ was considered to indicate a statistically significantly difference vs. NC group. Images were analyzed by Image-Pro Plus 6.0 software. CTD, cantharidin, NC, negative control; LC3 I, microtubule-associated proteins 1A/1B light chain 3A.

significantly reduced following treatment with $1 \mu \mathrm{M}$ CTD. The corresponding cell numbers for A549 cells were $86 \pm 6$ and $29 \pm 2$ in the NC and CTD groups, respectively (Fig. 2B, P<0.05), indicating the obstruction of invasive ability of A549 cells by CTD. A migration assay reveled similar results to the invasion assay, as the number of migrated A549 cells was also significantly suppressed following CTD treatment $(18 \pm 5)$, when compared with the NC groups $(32 \pm 2)$ (Fig. $2 \mathrm{C}$ and $\mathrm{D} ; \mathrm{P}<0.05$ ). 
A

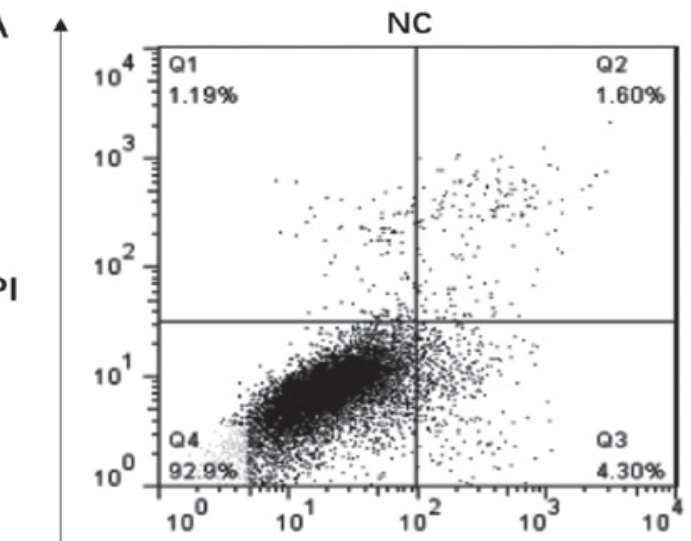

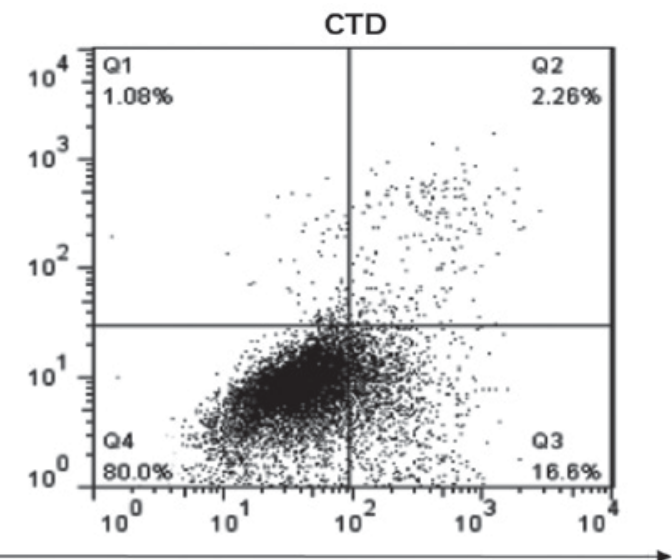

Annexin V-FITC
B

Bcl-2

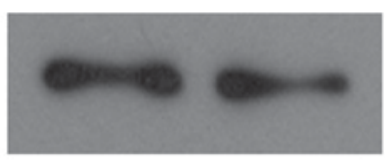

Bax

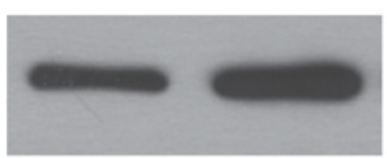

\section{Active \\ caspase-3}

GAPDH
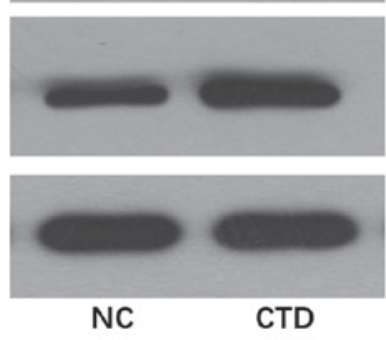
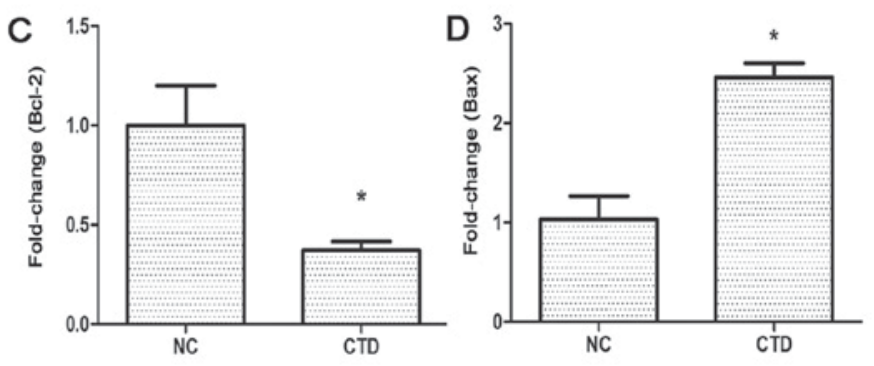

E

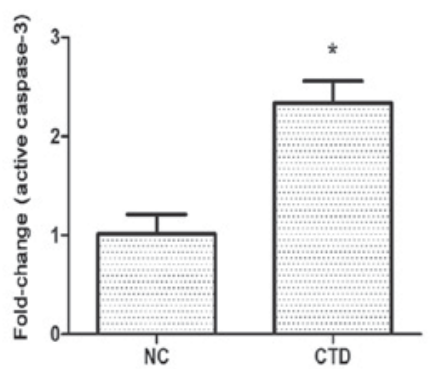

Figure 4. CTD induces apoptosis in A549 cells. (A) Flow cytometric analysis of A549 cell apoptosis by double staining with Annexin V-FITC and propidium iodide (PI). (B) The protein expression of Bax, Bcl-2, active caspase-3 were determined by western blotting. The protein expression of GAPDH was as used as the internal control. The protein expressions were quantified for (C) Bcl-2, (D) Bax and (E) active caspase-3. Images were analyzed by Image-Pro Plus 6.0 software. Results are presented as the mean \pm standard deviation of three independent experiments. ${ }^{*} \mathrm{P}<0.05$ was considered to indicate a statistically significantly difference vs. NC groups. Bcl-2, B-cell lymphoma-2; Bax, Bcl-associated X; CTD, cantharidin; FITC, fluorescein isothiocyanate; NC, negative control.

CTD promotes A549 cell autophagy. To investigate whether there was an association between autophagy and the effects of CTD in A549 cells, western blotting was performed. The results demonstrated that there was a significant increase in the expression of LC3 I/LC3 II ( $<2.3$-fold) and Beclin-1 ( $<2.5$-fold), and a decrease in the expression of p62 in CTD-treated A549 cells ( $<0.3$-fold), when compared with the control group (Fig. 3; $\mathrm{P}<0.05$ ). These results indicated that autophagy may be involved in the inhibiting role of CTD in A549 cell migration and growth.

CTD induces A549 cell apoptosis. To demonstrate the role of CTD in apoptosis further, A549 cells were treated with $1 \mu \mathrm{M}$ CTD and underwent flow cytometry analysis using annexin-V/PI. As demonstrated in Fig. 4A, the proportion of A549 cells undergoing apoptosis significantly increased in the CTD-treated group $(18.8 \pm 0.5 \%)$ compared with the NC group $(5.9 \pm 0.3 \%)(\mathrm{P}<0.05)$. Western blot analysis was then used to detect any changes in the levels of apoptosis-associated proteins. The results demonstrated that the expression of Bcl-2 was significantly decreased $(<0.4$-fold) in the CTD-treated groups (Fig. 4B and C; $\mathrm{P}<0.05$ ), whereas the expression level of Bax was upregulated ( $<2.5$-fold; Fig. $4 \mathrm{~B}$ and $\mathrm{D} ; \mathrm{P}<0.05)$. The level of active caspase-3, a paramount cleavage enzyme associated with the intrinsic and extrinsic apoptosis pathways, was also measured by western blotting. The results demonstrated that the expression level of active caspase- 3 was increased ( $<2.2$-fold) following CTD treatment (Fig. 4B and E; P<0.05). Taken together these results indicated that CTD may activate apoptotic pathways in A549 cells.

CTD inhibits activity of phosphatidylinositol 3-kinase (PI3K) signaling in A549 cells. Previous studies have demonstrated that the PI3K/Akt/mTOR pathway has a marked effect on tumor growth and survival $(16,17)$. To confirm whether the $\mathrm{PI} 3 \mathrm{~K} / \mathrm{Akt} / \mathrm{mTOR}$ pathway was associated with the role of CTD in A549 cells, the present study examined the changes in the levels of total and p-Akt, and p-mTOR (Fig. 5A-E). 

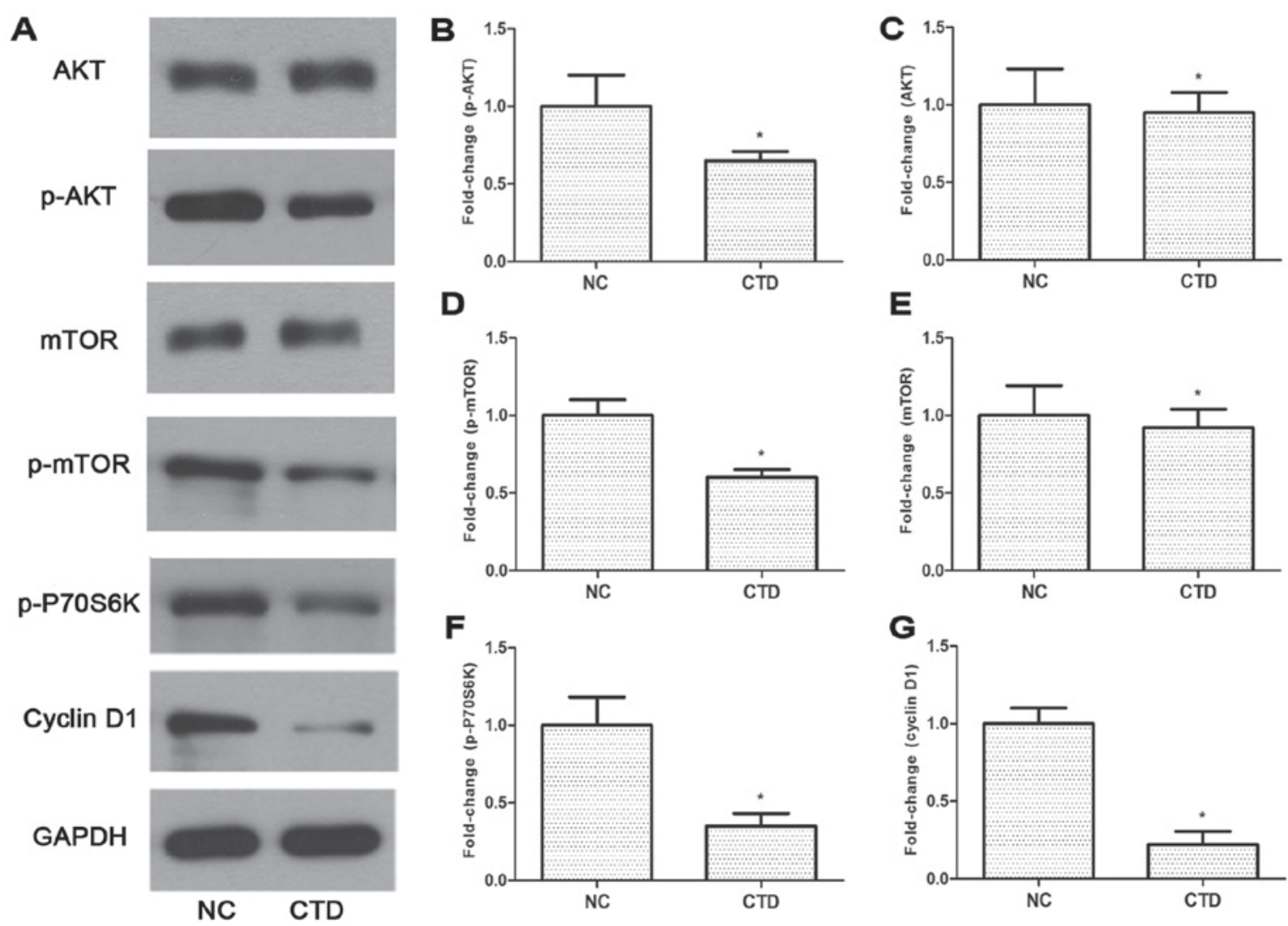

Figure 5. Effect of cantharidin on the relative protein levels of PI3K/Akt/mTOR signaling molecules in A549 cells. (A-G) Western blotting detected proteins involved in the PI3K/Akt/mTOR signaling pathway, including expression (B) p-Akt, (C) Akt, (D) mTOR, (E) p-mTOR, (F) p-p70S6K and (G) cyclin D1. "P<0.05, compared with NC groups. PI3K, phosphoinositide 3-kinase; p-Akt, phosphorylated RAC serine/threonine protein kinase; mTOR, mechanistic target of rapamycin; p70S6K, p7-S6 kinase; CTD, cantharidin; NC, negative control.

The downstream factors p-p70S6K and cyclin D1 of the $\mathrm{PI} 3 \mathrm{~K} / \mathrm{Akt} / \mathrm{mTOR}$ pathway were also examined (Fig. 5F and $5 \mathrm{G})$. Treatment with CTD led to the markedly decreased expression of p-Akt $(<0.7$-fold $)$, p-mTOR $(<0.6$-fold $)$, p-p70S6K ( $<0.3$-fold) and cyclin D1 ( $<0.2$-fold) levels in A549 cells (Fig 5B, D and G). These results demonstrated that CTD might inhibit the proliferation and metastasis of A549 cells via the PI3K/AKT/mTOR signaling pathway.

\section{Discussion}

In the present study, CTD was identified to significantly suppress the proliferation, migration and invasion of A549 cells. It was also observed that CTD may potentiate A549 cell autophagy and apoptosis. Furthermore, the present study provided evidence to indicate that CTD may exert its effects via inhibition of the PI3K/Akt/mTOR pathway.

Previous studies have reported that CTD efficiently inhibited proliferation and induced apoptosis in various cancer cells, including oral squamous cell carcinoma, renal carcinoma and gastric cancer cells (18-20). The results of the present study also demonstrated that CTD inhibited A549 cell proliferation and migration, which is consistent with the results of previous studies (18-20). The combined results of these studies provide evidence that CTD is a promising therapeutic candidate for the treatment of NSCLC.

A number of studies have reported that CTD exerted an inhibitory effect on cell proliferation by inducing apoptosis in a variety of tumor cells, including gastric, colorectal and pancreatic cancer cell lines (20-22). The results of the present study also demonstrated that CTD had a similar effect in promoting the apoptosis of NSCLC cells, by detecting levels of apoptotic markers, including anti-apoptosis protein $\mathrm{Bcl}-2$ and pro-apoptosis proteins like Bax and active caspase-3. Based on the background that autophagy is a survival-promoting process that captures, degrades, and recycles intracellular constituents in lysosomes and is considered to serve a distinct role in the suppression of tumorigenesis and promotion of mortality $(23,24)$, to investigate the underlying mechanism, autophagy markers were detected, namely, LC3, p62 and Beclin 1. The results revealed that CTD also exhibited an ability to induce autophagy. Previous studies have demonstrated that autophagy is able to accelerate the incidence of apoptosis and suppress tumor cell multiplication and growth $(25,26)$, which is line with the present results. The pathways involved in autophagy progression are complicated. The PI3K/Akt/mTOR signaling pathway is closely associated with tumor growth and survival, was implicated to be involved in suppressing autophagy (26). It is established that PI3K is regarded as a key regulator in various essential cellular processes, including cell survival, growth, and differentiation. Once PI3K is activated, its catalytic subunit activates AKT by phosphorylating AKT and successively activates mTOR by phosphorylating mTOR. The activation of mTOR leads to phosphorylation of p70S6K1, a mediator of protein translation and cell growth (27-29). It has also been demonstrated that Cyclin D1, a PI3K/AKT pathway 
downstream factor, is associated with abnormal proliferation, invasion, and thus prognosis of cancer (30). In the present study, western blot analysis revealed that the PI3K/Akt/mTOR signaling pathway was inhibited in CTD-treated A549 cells, which may have enhanced autophagy in addition to its induction of apoptosis. However, there remained certain limitations of the study. Only one cell line and concentration of CTD were examined and no animal models were used. Future studies may focus on using an expanded variety of cell lines, or developing animal models.

In conclusion, the results of the present study indicate that CTD impeded cell growth and migration by promoting autophagy and apoptosis, which may be regulated by inhibiting the PI3K/Akt/mTOR signaling pathway in NSCLC. Therefore, this work provides a novel insight that CTD may serve as a potential candidate for development of a naturally derived antitumor agent.

\section{References}

1. Siegel R, DeSantis C, Virgo K, Stein K, Mariotto A, Smith T, Cooper D, Gansler T, Lerro C, Fedewa S, et al: Cancer treatment and survivorship statistics. CA Cancer J Clin 64: 220-241, 2012.

2. Blackhall $\mathrm{F}$ and Thatcher N: Chemotherapy for advanced lung cancer. Eur J Cancer 40: 2345-2348, 2004.

3. Grilli R, Oxman AD and Julian JA: Chemotherapy for advanced non-small-cell lung cancer: How much benefit is enough? J Clin Oncol 11: 1866-1872, 1993

4. Iwamoto T: Clinical application of drug delivery systems in cancer chemotherapy: Review of the efficacy and side effects of approved drugs. Biol Pharm Bull 36: 715-718, 2013.

5. Olaussen KA and Postel-Vinay S: Predictors of chemotherapy efficacy in non-small-cell lung cancer: A challenging landscape. Ann Oncol 27: 2004-2016, 2016.

6. Yamamoto Y and Iwase H: [Management for treatment-induced adverse reaction-chemotherapy]. Nihon Rinsho 70 (Suppl 7): S672-S676, 2012.

7. Rabinowitz JD and White E: Autophagy and metabolism Science 330: 1344-1348, 2010

8. Duffy A, Le J, Sausville E and Emadi A: Autophagy modulation: A target for cancer treatment development. Cancer Chemother Pharmacol 75: 439-447, 2015.

9. Li C, Wang Y, Wang C, Yi X, Li M and He X: Anticancer activities of harmine by inducing a pro-death autophagy and apoptosis in human gastric cancer cells. Phytomedicine 28: 10-18, 2017.

10. Wang B, Lu D, Xuan M and Hu W: Antitumor effect of sunitinib in human prostate cancer cells functions via autophagy. Exp Ther Med 13: 1285-1294, 2017.

11. White E: The role for autophagy in cancer. J Clin Invest 125: 42-46, 2015.

12. Parzych KR and Klionsky DJ: An overview of autophagy: Morphology, mechanism, and regulation. Antioxid Redox Signal 20: 460-473, 2014
13. Xiong X, Wu M, Zhang H, Li J, Lu B, Guo Y, Zhou T, Guo H, Peng R, Li X, et al: Atg5 siRNA inhibits autophagy and enhances norcantharidin-induced apoptosis in hepatocellular carcinoma. Int J Oncol 47: 1321-1328, 2015.

14. Kim YM, Ku MJ, Son YJ, Yun JM, Kim SH and Lee SY: Anti-metastatic effect of cantharidin in A549 human lung cancer cells. Arch Pharm Res 36: 479-484, 2013.

15. Zhang WD, Zhao HR, Yan Y, Wang XH, Zong ZH and Liu Y: Apoptosis induced by cantharidin in human pulmonary carcinoma cells A549 and its molecular mechanisms. Zhonghua Zhong Liu Za Zhi 27: 330-334, 2005 (In Chinese).

16. Vanhaesebroeck B, Leevers SJ, Panayotou G and Waterfield MD: Phosphoinositide 3-kinases: A conserved family of signal transducers. Trends Biochem Sci 22: 267-272, 1997.

17. Pellegatta F, Chierchia SL and Zocchi MR: Functional association of platelet endothelial cell adhesion molecule-1 and phosphoinositide 3-kinase in human neutrophils. J Biol Chem 273: 27768-27771, 1998.

18. Ren Y, Zhang SW, Xie ZH, Xu XM, Chen LL, Lou ZG, Weng GB and Yao XP: Cantharidin induces G2/M arrest and triggers apoptosis in renal cell carcinoma. Mol Med Rep 14: 5614-5618, 2016.

19. Su CC, Lee KI, Chen MK, Kuo CY, Tang CH and Liu SH: Cantharidin induced oral squamous cell carcinoma cell apoptosis via the JNK-regulated mitochondria and endoplasmic reticulum stress-related signaling pathways. PLoS One 11: e0168095, 2016.

20. Zhang C, Chen Z, Zhou X, Xu W, Wang G, Tang X, Luo L, Tu J, Zhu Y, Hu W, et al: Cantharidin induces G2/M phase arrest and apoptosis in human gastric cancer SGC-7901 and BGC-823 cells. Oncol Lett 8: 2721-2726, 2014

21. Liu B, Gao HC, Xu JW, Cao H, Fang XD, Gao HM and Qiao SX: Apoptosis of colorectal cancer UTC116 cells induced by Cantharidinate. Asian Pac J Cancer Prev 13: 3705-3708, 2012.

22. Li W, Xie L, Chen Z, Zhu Y, Sun Y, Miao Y, Xu Z and Han X: Cantharidin, a potent and selective PP2A inhibitor, induces an oxidative stress-independent growth inhibition of pancreatic cancer cells through G2/M cell-cycle arrest and apoptosis. Cancer Sci 101: 1226-1233, 2010.

23. Anding AL and Baehrecke EH: Autophagy in cell life and cell death. Curr Top Dev Biol 114: 67-91, 2015.

24. Denton D, Xu T and Kumar S: Autophagy as a pro-death pathway. Immunol Cell Biol 93: 35-42, 2015.

25. Wang YX, Xu SQ, Chen XH, Liu RS and Liang ZQ: Autophagy involvement in olanzapine-mediated cytotoxic effects in human glioma cells. Asian Pac J Cancer Prev 15: 8107-8113, 2014.

26. Mihalache CC, Yousefi S, Conus S, Villiger PM, Schneider EM and Simon HU: Inflammation-associated autophagy-related programmed necrotic death of human neutrophils characterized by organelle fusion events. J Immunol 186: 6532-6542, 2011.

27. Hassan B, Akcakanat A, Holder AM and Meric-Bernstam F: Targeting the PI3-kinase/Akt/mTOR signaling pathway. Surg Oncol Clin N Am 22: 641-664, 2013.

28. Sheppard K, Kinross KM, Solomon B, Pearson RB and Phillips WA: Targeting PI3 kinase/AKT/mTOR signaling in cancer. Crit Rev Oncog 17: 69-95, 2012.

29. Matsuoka T and Yashiro M: The role of PI3K/Akt/mTOR signaling in gastric carcinoma. Cancers (Basel) 6: 1441-1463, 2014.

30. Alao JP: The regulation of cyclin D1 degradation: Roles in cancer development and the potential for therapeutic invention. Mol Cancer 6: 24, 2007. 